\title{
Endoscopic management of Barrett's and early oesophageal neoplasia
}

\author{
G Lipman, ${ }^{1,2}$ RJ Haidry ${ }^{1,2}$
}

\begin{abstract}
${ }^{1}$ Department of
Gastroenterology, University College Hospital NHS

Foundation Trust, London, UK

${ }^{2}$ Division of Surgery \&

Interventional Science, University

College London, London, UK
\end{abstract}

\section{Correspondence to}

RJ Haidry, Department of Gastroenterology, University College London, Cruciform Building, Gower Street, London WC1E 6B, UK; rehan.haidry@uclh.nhs.uk

Received 25 October 2016 Revised 19 December 2016 Accepted 26 December 2016

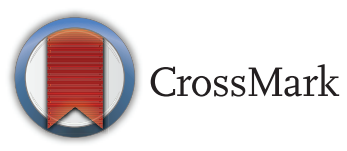

To cite: Lipman G, Haidry RJ. Frontline Gastroenterology 2017;8:138-142.

\begin{abstract}
Barrett's oesophagus (BO) is the only known precursor to oesophageal adenocarcinoma (OAC). Dysplasia and intramucosal cancer arising in BO can safely be treated with endoscopic eradication therapy (EET) due to the low risk of subsequent lymph node metastasis. Treatment at an early stage is paramount due to the ongoing poor prognosis and outcomes of patients with advanced OAC. The mainstay of treatment is endoscopic resection of visible lesions for accurate staging followed by ablation therapy to all remaining columnar-lined epithelium, most commonly with radiofrequency ablation.

Successful eradication of dysplasia can be achieved in $>95 \%$ of patients with this EET combined approach.
\end{abstract}

\section{BACKGROUND}

The UK has one of the highest incidence of oesophageal adenocarcinoma (OAC) in the world. ${ }^{1}$ OAC has a poor prognosis, with $<20 \%$ of patients surviving at 5 years. $^{2}$ An important factor driving this poor survival is the late presentation of the disease.

The only known precursor to $\mathrm{OAC}$ is Barrett's oesophagus (BO). The overall risk of $\mathrm{BO}$ to progression to OAC is estimated at $0.12 \%-0.48 \%$ per year, while the risk for progression to OAC or highgrade dysplasia (HGD) is $0.26 \%-0.63 \%$ per year. ${ }^{3-7}$ Progression of low-grade dysplasia (LGD) to OAC has been reported as 5.1 per 1000 patient years compared with 1 per 1000 patient years without dysplasia. ${ }^{5} \mathrm{HGD}$ and intramucosal cancer (IMC) has a reported risk of OAC of over $10 \%$ per year. $^{8}$

\section{Risk factors}

Risk factors for progression to HGD or OAC include reflux, ${ }^{9}$ male sex, ${ }^{10}$ increasing age, ${ }^{7}$ obesity (particularly waist:hip ratio) ${ }^{11}$ and length of $\mathrm{BO}^{12}$

\section{The metaplasia, dysplasia, cancer pathway}

Chronic exposure to acid or bile refluxate injures the epithelium resulting in inflammation and cell proliferation. In combination with genetic instability, this is thought to trigger the development of Barrett's metaplasia. Continued exposure and inflammation activates epithelial-mesenchymal transition to the development of LGD, HGD and finally invasive OAC. ${ }^{13}$

\section{Staging of dysplasia/early cancer}

Staging of early oesophageal cancers allows the stratification of patients according to subsequent lymph node metastatic risk and allows appropriate local modalities of treatment to be offered. Once a tumour involves regional nodes or distant metastases, local treatment with endoscopic intervention is no longer a curative option. It is therefore imperative in patients with Barrett's dysplasia or early OAC that treatment is offered when there is still curative intent. The depth of the cancer is correlated with the risk of lymph node metastases. ${ }^{14}$

IMC also known as a T1a cancer carries a $1.3 \%$ risk of lymph node metastasis, ${ }^{15}$ whereas the risk with submucosal $\mathrm{T} 1 \mathrm{~b}$ cancer is related to the depth of invasion; ${ }^{16}$ with SM1 carrying a $6 \%$ risk, SM2 a 23\% risk and SM3 a 58\% risk. ${ }^{17}$

Further risk factors for lymph node involvement include histological evidence of poorly differentiated tumour grade and lymphovascular invasion. ${ }^{17}$ Due to this risk of nodal disease, only LGD, HGD and tumours staged as T1a, or T1b with no poor prognostic markers are offered endoscopic treatment. ${ }^{18}$ Recent National Oesophago-Gastric Cancer Audit (NOCGA) data indicate the risk of surgical mortality following oesophagectomy is higher than the risk of lymph node metastases in patients with 
superficial cancer and HGD; ${ }^{19}$ hence, the British Society of Gastroenterology (BSG) Guidelines currently recommend endoscopic eradication therapy (EET) as first-line therapy for early disease. ${ }^{18}$

\section{DYSPLASIA DETECTION}

Early detection of lesions in $\mathrm{BO}$ is essential to drive any subsequent endoscopic treatment options. Visualisation of subtle mucosal lesions can be enhanced with novel wide-field imaging technology that can be used on the entire oesophagus to highlight areas of interest including acetic acid chromoendoscopy and virtual chromoendoscopy (summarised in table 1). Both narrow band imaging (NBI) and i-Scan technologies can be combined with magnification endoscopy, which allow areas of interest to be viewed with up 136 times magnification.

Acetic acid (2\%-3\% strength) causes the metaplastic (Barrett's) epithelium to whiten, highlighting the mucosal pattern. Dysplastic lesions are thought to lose this white appearance faster than non-dysplastic areas, permitting targeted assessment and sampling of these areas (figure 1). A recent meta-analysis of nine studies, calculated a pooled sensitivity of $92 \%$ and specificity of $96 \%$ for the diagnosis of high-grade dysplasia or IMC. ${ }^{20}$

Real-time in vivo diagnosis of Barrett's dysplasia is possible with confocal laser endomicroscopy but requires a targeted approach and operator expertise for interpretation. ${ }^{21}$

Although all these technologies allow for greater detection of subtle lesions, they also require specialist expertise to recognise these abnormalities. Recent research has shown that a longer procedure time to allow a detailed inspection of the mucosa using highquality white light imaging may yield just as accurate diagnosis. ${ }^{22}$

\section{TREATMENT OPTIONS}

Historically, treatment for early gastrointestinal neoplasm required major surgery, with the associated morbidity and mortality of oesophagectomy. Early neoplasms confined to the superficial layers of the

Table 1 Virtual chromoendoscopy technologies

\begin{tabular}{ll}
\hline Technology & Mechanism \\
\hline $\begin{array}{l}\text { Narrow Band Imaging Olympus } \\
\text { Medical Systems (Olympus, } \\
\text { Japan) }\end{array}$ & $\begin{array}{l}\text { Optic filtering of reflected light from } \\
\text { the mucosa, isolating blue (415 nm) } \\
\text { and green }(540 \mathrm{~nm}) \text { bands of light to } \\
\text { enhance visualisation. }\end{array}$ \\
$\begin{array}{l}\text { i-Scan and i-Scan OE PENTAX } \\
\text { (HOYA, Japan) }\end{array}$ & $\begin{array}{l}\text { Digital postprocessing image } \\
\text { enhancement to improve mucosal } \\
\text { pattern and vascularity. New OE } \\
\text { technology combines this with optic } \\
\text { filtering. } \\
\text { FICE (Fujinon, Japan) }\end{array}$ \\
& $\begin{array}{l}\text { Postprocessing technology that } \\
\text { reconstructs a virtual image of a } \\
\text { single wavelength in real time. }\end{array}$ \\
\hline
\end{tabular}

mucosa and submucosa can now be treated endoscopically with minimal risk of nodal and metastatic spread. ${ }^{17}$ Despite these advances and BSG guidelines, the 2015 NOCGA reported that of 930 patients with HGD, 26.2\% underwent surveillance alone with no therapy, $67.5 \%$ received endoscopic treatment and $6.3 \%$ surgical resection. ${ }^{19}$

\section{Endoscopic mucosal resection}

Endoscopic mucosal resection (EMR) enables accurate histological assessment of the depth of invasion of early neoplastic lesions, thus being both a diagnostic and a therapeutic intervention. EMR can both upstage the diagnosis in $10 \%$ of patients and downgrade the diagnosis in $21 \% .^{23}$ EMR techniques include cap-assisted, ligation-assisted (the Duette multiband mucosectomy device, Cook, USA or the Captivator device, Boston Scientific, USA) and cap- and snare-assisted resection. As monotherapy, complete resolution of dysplasia (CR-D) rates are 87\%-96\%, but strictures occur in 12.5\%-88\% (higher rates with larger and circumferential resections). ${ }^{24} 25$

\section{Endoscopic submucosal dissection}

In contrast to EMR, where large lesions may be removed piecemeal, endoscopic submucosal dissection (ESD) allows large lesions to be removed en bloc, as well as resection of tumours arising from the muscularis propria. ESD requires extensive training, longer procedure time and is associated with an increased risk of bleeding and perforation. ${ }^{26}$ There is also an increased rate of stricture formation after ESD which can make subsequent ablation of the residual BO area challenging. ${ }^{27}$ Therefore, ESD is only considered for lesions larger than $15 \mathrm{~mm}$, poorly lifting tumours and lesions at risk of submucosal invasion. ${ }^{28}$

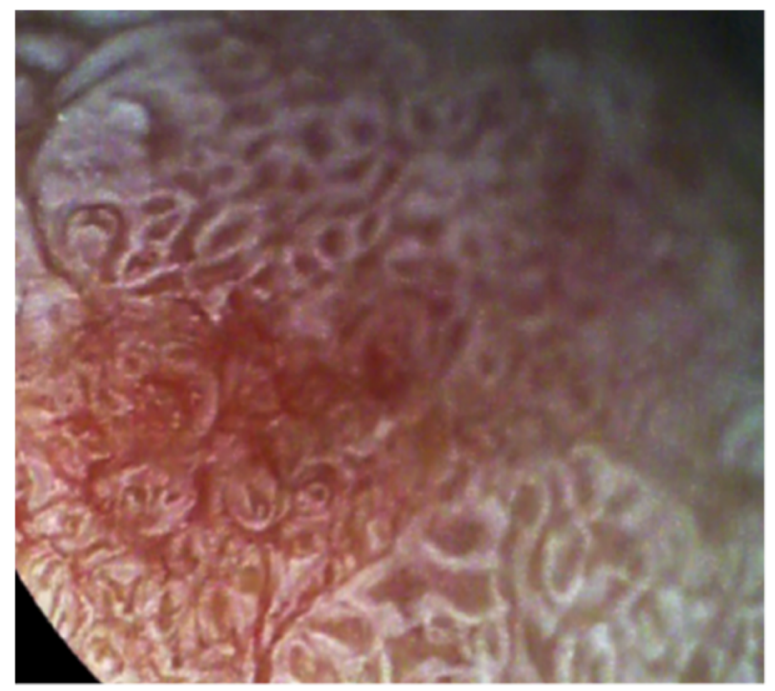

Figure 1 i-Scan magnification endoscopy to demonstrate the loss of whitening after application of $2 \%$ acetic acid in dysplastic Barrett's oesophagus. 


\section{Radiofrequency ablation}

Radiofrequency ablation (RFA) (Medtronic) is a field ablation technique used to ablate the surface $500 \mu \mathrm{m}$ of the gastrointestinal mucosa. It is primarily used in the treatment of Barrett's-related neoplasia using a balloon (to provide a $360^{\circ}$ ablation field) or a focal device, mounted over the endoscope (figure 2). Several treatments over the course of months are required to effectively treat $\mathrm{BO}$ and any associated neoplasia. The treatment has a high success rates and good durability data now exist and has over the past 10 years become the mainstay of treatment of these patients with BO neoplasia. $^{29}$

The breakthrough randomised controlled trial in 2009 reported CR-D rates of $81 \%$ of patients with HGD with a $6 \%$ stricture rate. ${ }^{30}$ However, RFA treatment alone is insufficient for the treatment of Barrett's dysplasia where there are nodules or ulcers due to the risk of buried dysplasia. Therefore, all visible lesions are first removed with EMR and the residual BO, whether dysplastic or only metaplastic is treated with RFA. ${ }^{18}$ The UK RFA Registry reports that this combined EMR and RFA approach achieves CR-D rates of $86 \%$ and complete resolution of intestinal metaplasia (CR-IM) rates of $62 \% .{ }^{31}$ Recently, a multicentre trial used this combined approach and allowed visible BO at the end of 12 months treatment to be retreated with either a further resection or an argon plasma coagulation (APC) treatment. This protocol achieved 92\% CR-D and $87 \%$ CR-IM with only $4 \%$ of patients reporting recurrence at 36 months of follow-up. ${ }^{32}$
Treatment of LGD is now recommended following the SURF trial that reported patients who received RFA had lower rates of progression to HGD or OAC than those receiving surveillance only. ${ }^{33}$

\section{Recurrence}

With all endoscopic therapies for Barrett's neoplasia, recurrence of disease following initially successful treatment remains a risk. RFA has been shown to be a durable treatment option for Barrett's-associated neoplasia with a recent meta-analysis reported a pooled recurrence rate of $6.0 \%$ per patient year. ${ }^{34}$ Following eradication of HGD by endoscopic therapy or surgery, endoscopic follow-up is required ${ }^{35}$ and recent data combining the US and UK RFA registries, has suggested follow-up intervals can be stratified according to risk. Most patients with HGD and IMC should have endoscopic examinations of the treated oesophagus 3, 9, 18 and 30 months after treatment ends and should continue thereafter with yearly assessments. ${ }^{36}$

\section{Specialised centres}

Reflecting the centralisation of upper gastrointestinal surgical centres for cancer in the UK, the BSG also recommends the centralisation of endoscopic resection due to the low but significant risk of complications requiring surgical intervention. As most patients undergoing treatment for BO-associated dysplasia require dual therapy with EMR and RFA, the BSG advises that RFA also be performed only in centres where endoscopic resection can be performed. The
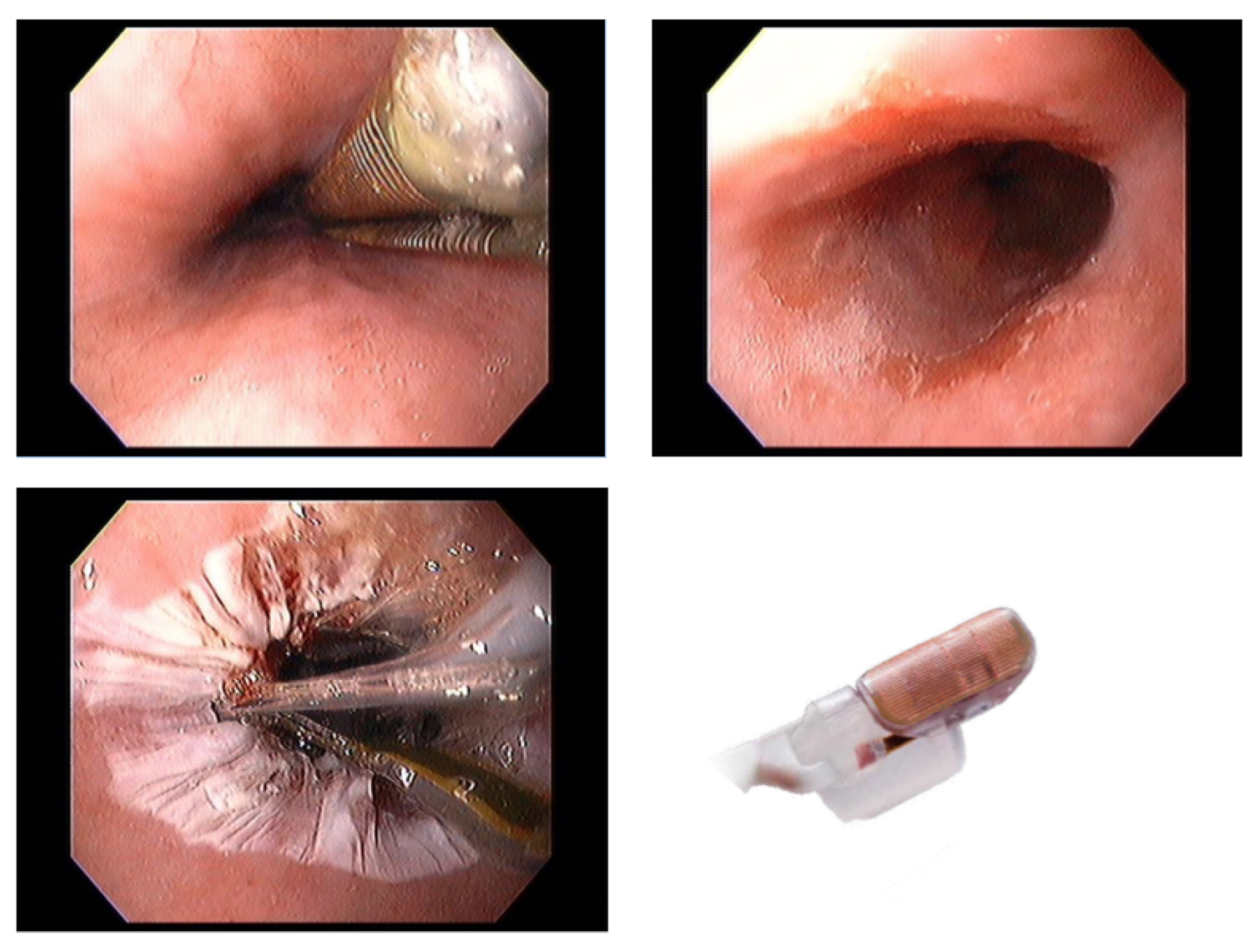

Figure 2 Radiofrequency ablation of Barrett's oesophagus. Circumferential Barrett's oesophagus prior to ablation (top left), placement of the 360 balloon catheter (top right) and following ablation (bottom left). Focal devices are also available (bottom right) (with permission from Medtronic). 
BSG also recommends that a minimum of 30 supervised cases of EMR and RFA should be performed to acquire the necessary technical skills and management of complications. ${ }^{18}$

\section{Argon plasma coagulation}

APC uses argon gas to conduct electrical current to thermally ablate targeted tissue. APC is often used as an adjunct to alternative therapies, as it is cheap and easy to treat small areas of residual disease and has been demonstrated to significantly increase recurrence-free survival for the patients undergoing ablation after EMR compared with those having EMR alone. ${ }^{37}$ A feasibility study comparing APC with RFA recently reported preliminary findings that suggested that at 12 months there was no difference in CR-D rates. ${ }^{38}$ However, there are still no large volume data to support APC as first-line ablative therapy for patients with BO neoplasia and further studies are required in this field.

\section{THE FUTURE}

The development of endoscopic tools to improve the diagnosis and management of patients with Barrett's dysplasia is on-going and evolving rapidly. Several technologies are currently in clinical trials to assess their efficacy.

\section{Imaging}

Autoflouresence (AF) is a virtual endoscopy technique using the variable quantities of fluorophores (substances that emit fluorescent light after exposure to short, blue light wavelengths). Alterations in the autofluorescence pattern of neoplastic tissue is translated into false colour images, usually depicting neoplasia in purple against a green background of healthy mucosa. $\mathrm{AF}$ has been integrated with high definition white light endoscopy and NBI as part of the 'endoscopic trimodal imaging' system.

Optical coherence tomography (OCT) uses reflected light in a manner similar to acoustic ultrasound to generate high-resolution three-dimensional images. This allows 'visualisation' of the mucosa to a depth of 1$2 \mathrm{~mm}$. Currently performed with a probe through the working channel of an endoscope, the future may involve tethered capsule technology (where capsules attached to a string are swallowed and then retrieved) and rapid assessment of the tubular oesophagus. Recent studies have suggested a role for OCT for surveillance after ablation therapy with radio-frequency ablation.

\section{Treatment}

Cryoablation uses liquid nitrogen to form intracellular and extracellular ice that causes ischaemic necrosis on thawing and apoptosis of the treated cells. Previous limitations of cryotherapy included the large volume of gas produced during therapy; however, a new focal balloon device avoids this risk using a self-contained unit that does not introduce gas into the stomach
(Cryoballoon Focal Ablation System, C2Therapeutics, USA).

A 2016 study reported on 96 patients, 91\% of patients with LGD achieved CR-D and 61\% CR-IM and $81 \%$ of those with HGD achieved CR-D and $65 \%$ CR-IM. No perforations or deaths were reported and there was a single stricture that did not require dilatation. ${ }^{39}$

\section{CONCLUSION}

The management of Barrett's-associated dysplasia has changed dramatically in the last 10 years. Our continued understanding of which lesions are amenable to EET means that we can confidently offer a long term curative treatment to the majority of patients with early disease and avoid disease progression and the need for surgery for these select patients. Meticulous lesion recognition with combined high-quality endoscopic imaging modalities followed by endoscopic resection and ablation with RFA remain the foundation of treatment for most patients. Advances in optical imaging for diagnostic and surveillance purposes coupled with new interventional technologies such as cryotherapy may further improve outcomes and allow us to treat a wider cohort of patients.

Contributors Both authors have contributed to the writing of this article.

Competing interests None declared.

Provenance and peer review Commissioned; externally peer reviewed.

\section{REFERENCES}

1 Cancer Research UK. Oesophageal cancer incidence statistics. 2013 (cited 1 August 2016). http://www.cancerresearchuk.org/ health-professional/cancer-statistics/statistics-by-cancer-type/ oesophageal-cancer/incidence\#ref-5

2 Pennathur A, Gibson MK, Jobe BA, et al. Oesophageal carcinoma. Lancet 2013;381:400-12.

3 Wani S, Falk G, Hall M, et al. Patients with nondysplastic Barrett's esophagus have low risks for developing dysplasia or esophageal adenocarcinoma. Clin Gastroenterol Hepatol 2011;9:220-7; quiz e26.

4 Bhat S, Coleman HG, Yousef F, et al. Risk of malignant progression in Barrett's Esophagus patients: results from a large population-based study. J Natl Cancer Inst 2011;103:1049-57.

5 Hvid-Jensen F, Pedersen L, Drewes AM, et al. Incidence of adenocarcinoma among patients with Barrett's esophagus. N Engl J Med 2011;365:1375-83.

6 Desai TK, Krishnan K, Samala N, et al. The incidence of oesophageal adenocarcinoma in non-dysplastic Barrett's oesophagus: a meta-analysis. Gut 2012;61:970-6.

7 de Jonge PJF, van Blankenstein M, Looman CWN, et al. Risk of malignant progression in patients with Barrett's oesophagus: a Dutch nationwide cohort study. Gut 2010;59:1030-6.

8 Weston AP, Sharma P, Topalovski M, et al. Long-term follow-up of Barrett's high-grade dysplasia. Am J Gastroenterol 2000;95:1888-93.

9 Thrift AP, Kramer JR, Qureshi Z, et al. Age at onset of GERD symptoms predicts risk of Barrett's esophagus. Am J Gastroenterol 2013;108:915-22. 
10 Coleman HG, Bhat SK, Murray LJ, et al. Symptoms and endoscopic features at Barrett's esophagus diagnosis: implications for neoplastic progression risk. Am J Gastroenterol 2014;109:527-34.

11 Di Caro S, Cheung WH, Fini L, et al. Role of body composition and metabolic profile in Barrett's oesophagus and progression to cancer. Eur J Gastroenterol Hepatol 2016;28:251-60.

12 Anaparthy R, Gaddam S, Kanakadandi V, et al. Association between length of Barrett's esophagus and risk of high-grade dysplasia or adenocarcinoma in patients without dysplasia. Clin Gastroenterol Hepatol 2013;11:1430-6.

13 Poehlmann A, Kuester D, Malfertheiner P, et al. Inflammation and Barrett's carcinogenesis. Pathol Res Pract 2012;208:269-80.

14 Shimada H, Nabeya Y, Matsubara H, et al. Prediction of lymph node status in patients with superficial esophageal carcinoma: analysis of 160 surgically resected cancers. Am J Surg 2006;191:250-4.

15 Leers JM, DeMeester SR, Oezcelik A, et al. The prevalence of lymph node metastases in patients with T1 esophageal adenocarcinoma a retrospective review of esophagectomy specimens. Ann Surg 2011;253:271-8.

16 Westerterp M, Koppert LB, Buskens CJ, et al. Outcome of surgical treatment for early adenocarcinoma of the esophagus or gastro-esophageal junction. Virchows Arch 2005;446:497-504.

17 Gockel I, Sgourakis G, Lyros O, et al. Risk of lymph node metastasis in submucosal esophageal cancer: a review of surgically resected patients. Expert Rev Gastroenterol Hepatol 2011;5:371-84.

18 Fitzgerald RC, di Pietro M, Ragunath K, et al. British Society of Gastroenterology guidelines on the diagnosis and management of Barrett's oesophagus. Gut 2014;63:7-42.

19 Chadwick G, Varagunam M, Groene O, et al. National oesophago-gastric cancer audit. Health and Social Care Information Centre, 2015.

20 Coletta M, Sami SS, Nachiappan A, et al. Acetic acid chromoendoscopy for the diagnosis of early neoplasia and specialized intestinal metaplasia in Barrett's esophagus: a meta-analysis. Gastrointest Endosc 2016;83:57-67.

21 Wu J, Pan YM, Wang TT, et al. Confocal laser endomicroscopy for detection of neoplasia in Barrett's esophagus: a meta-analysis. Dis Esophagus 2014;27:248-54.

22 Gupta N, Gaddam S, Wani SB, et al. Longer inspection time is associated with increased detection of high-grade dysplasia and esophageal adenocarcinoma in Barrett's esophagus. Gastrointestinal Endoscopy 2012;76:531-8.

23 Wani S, Abrams J, Edmundowicz SA, et al. Endoscopic mucosal resection results in change of histologic diagnosis in Barrett's esophagus patients with visible and flat neoplasia: a multicenter cohort study. Dig Dis Sci 2013;58:1703-9.

24 Chennat J, Konda VJA, Ross AS, et al. Complete Barrett's eradication endoscopic mucosal resection: an effective treatment modality for high-grade dysplasia and intramucosal carcinoma-an American single-center experience. Am J Gastroenterol 2009;104:2684-92.

25 Larghi A, Lightdale CJ, Ross AS, et al. Long-term follow-up of complete Barrett's eradication endoscopic mucosal resection (CBE-EMR) for the treatment of high grade dysplasia and intramucosal carcinoma. Endoscopy 2007;39:1086-91.
26 Cao Y, Liao C, Tan A, et al. Meta-analysis of endoscopic submucosal dissection versus endoscopic mucosal resection for tumors of the gastrointestinal tract. Endoscopy 2009;41:751-7.

27 Lewis JJ, Rubenstein JH, Singal AG, et al. Factors associated with esophageal stricture formation after endoscopic mucosal resection for neoplastic Barrett's esophagus. Gastrointest Endosc 2011;74:753-60.

28 Pimentel-Nunes P, Dinis-Ribeiro M, Ponchon T, et al. Endoscopic submucosal dissection: European Society of Gastrointestinal Endoscopy (ESGE) Guideline. Endoscopy 2015;47:829-54.

29 Haidry RJ, Butt MA, Dunn JM, et al. Improvement over time in outcomes for patients undergoing endoscopic therapy for Barrett's oesophagus-related neoplasia: 6-year experience from the first 500 patients treated in the UK patient registry. Gut 2015;64:1192-9.

30 Shaheen NJ, Sharma P, Overholt BF, et al. Radiofrequency ablation in Barrett's esophagus with dysplasia TL-360.

N Engl J Med 2009;360:2277-88.

31 Haidry RJ, Dunn JM, Butt MA, et al. Radiofrequency ablation and endoscopic mucosal resection for dysplastic Barrett's esophagus and early esophageal adenocarcinoma: outcomes of the UK national halo RFA registry. Gastroenterology 2013;145:87-95.

32 Phoa KN, Pouw RE, Bisschops R, et al. Multimodality endoscopic eradication for neoplastic Barrett oesophagus: results of an European multicentre study (EURO-II). Gut 2016;65:555-62.

33 Phoa KN, van Vilsteren FGI, Weusten BL, et al. Radiofrequency ablation vs endoscopic surveillance for patients with Barrett esophagus and low-grade dysplasia. JAMA 2014;311:1209-17.

34 Krishnamoorthi R, Singh S, Ragunathan K, et al. Risk of recurrence of Barrett's esophagus after successful endoscopic therapy: a systematic review and meta-analysis. Gastrointest Endosc 2016;83:1090-106.e3.

35 Bennett C, Vakil N, Bergman J, et al. Consensus statements for management of Barrett's dysplasia and early-stage esophageal adenocarcinoma, based on a Delphi process. Gastroenterology 2012;143:336-46.

36 Cotton CC, Haidry R, Eluri S, et al. 887 evidence-based surveillance intervals following Radiofrequency Ablation (RFA) of Barrett's Esophagus (BE): an analysis of recurrence in the US RFA registry with validation in the UK National Halo Registry. Gastrointest Endosc 2016;83:AB181.

37 Manner H, Rabenstein T, Pech O, et al. Ablation of residual Barrett's epithelium after endoscopic resection: a randomized long-term follow-up study of argon plasma coagulation vssurveillance (APE study). Endoscopy 2014;46:6-12.

38 Peerally MF, Barr H, Lovat L, et al. 566 BRIDE (Barrett's Randomised Intervention for Dysplasia by Endoscopy) -results of a feasibility study comparing Argon Plasma Coagulation (APC) with Radiofrequency Ablation (RFA) after endoscopic resection of patients with high grade Dysplasia or T1 Ade. Gastrointest Endosc 2016;83:AB151.

39 Ghorbani S, Tsai FC, Greenwald BD, et al. Safety and efficacy of endoscopic spray cryotherapy for Barrett's dysplasia: results of the National Cryospray Registry. Dis Esophagus 2016;29:241-7. 